\title{
A Genetic Toolkit for Investigating Clavibacter Species: Markerless Deletion, Permissive Site Identification, and an Integrative Plasmid
}

\author{
Danielle M. Stevens, ${ }^{1,2}$ Andrea Tang, ${ }^{2}$ and Gitta Coaker ${ }^{2, \dagger}$ \\ ${ }^{1}$ Integrative Genetics and Genomics Graduate Group, University of California, Davis, Davis, CA 95616, U.S.A. \\ ${ }^{2}$ Department of Plant Pathology, University of California, Davis, Davis, CA 95616, U.S.A.
}

Accepted 7 September 2021.

\begin{abstract}
The development of knockout mutants and expression variants are critical for understanding genotype-phenotype relationships. However, advances in these techniques in gram-positive actinobacteria have stagnated over the last decade. Actinobacteria in the Clavibacter genus are composed of diverse crop pathogens that cause a variety of wilt and cankering diseases. Here, we present a suite of tools for genetic manipulation in the tomato pathogen Clavibacter michiganensis including a markerless deletion system, an integrative plasmid, and an $R$ package for identification of permissive sites for plasmid integration. The vector pSelAct-KO is a recombination-based, markerless knockout system that uses dual selection to engineer seamless deletions of a region of interest, providing opportunities for repeated higher-order genetic knockouts. The efficacy of pSelAct-KO was demonstrated in $C$. michiganensis and was confirmed using whole-genome sequencing. We developed permissR, an $R$ package to identify permissive sites for chromosomal integration, which can be used in conjunction with pSelActExpress, a nonreplicating integrative plasmid that enables recombination into a permissive genomic location. Expression of enhanced green fluorescent protein by pSelAct-Express was verified in two candidate permissive regions predicted by permissR in $C$. michiganensis. These molecular tools are essential advances for investigating gram-positive actinobacteria, particularly for important pathogens in the Clavibacter genus.
\end{abstract}

Keywords: actinobacteria, Clavibacter, permissive expression, unmarked knockouts

Gram-positive actinobacteria in the Clavibacter genus are economically important xylem-colonizing bacterial pathogens that can infect both monocots and dicots, resulting in canker and

${ }^{\dagger}$ Corresponding author: G. Coaker; glcoaker@ucdavis.edu

Funding: This research was supported by National Institute of Health 1R35GM136402 to G. Coaker and the United States Department of Agriculture National Institute of Food and Agriculture 2021-67034-35049 to D. M. Stevens.

*The $\boldsymbol{e}$-Xtra logo stands for "electronic extra" and indicates there are supplementary figures and tables published online.

The author(s) declare no conflict of interest.

(cc) (i) $(2)($ Copyright $(\odot 2021$ The Author(s). This is an open access article distributed under the CC BY-NC-ND 4.0 International license. wilting diseases (Eichenlaub and Gartemann 2011; Thapa et al. 2019). Despite current disease control measures including good horticultural practices and monitoring seed stock, outbreaks of Clavibacter pathogens have occurred in recent years (PeritoreGalve et al. 2021). The corn pathogen Clavibacter nebraskensis has been problematic for many corn-producing Midwestern states and the tomato pathogen $C$. michiganensis has caused notable losses in years past (Ahmad et al. 2015; Nandi et al. 2018; Peritore-Galve et al. 2021). While outbreaks are sporadic, four of the six species are considered quarantine organisms by the European and Mediterranean Plant Protection Organization, with C. michiganensis acting as a potential threat to both greenhouse and field production (Nandi et al. 2018; Peritore-Galve et al. 2021). Despite their agricultural impact, little is known about how these pathogens cause disease (Nandi et al. 2018; Thapa et al. 2019).

In other bacterial pathogens, genes important for pathogenicity and host range include secreted protein effectors that act either in the apoplast or inside cells to suppress host immunity, alter host metabolism, and enable colonization, providing a fitness advantage. Bacterial effectors are released by a variety of secretion systems including type II, III, IV, and V as well as Sec and Tat (Dalio et al. 2018; Ponciano et al. 2003). The most well-studied Clavibacter species is the tomato pathogen $C$. michiganensis. $C$. michiganensis carries effectors on two plasmids, pCM1 and pCM2, and within the approximately $129-\mathrm{kb}$ chp/tomA pathogenicity island (PAI) (Meletzus et al. 1993). While the entire chp/tomA PAI is only found in C. michiganensis, fragments or homologs of PAI members, or both, can be found in the genomes of other pathogenic Clavibacter species (Bentley et al. 2008; Hwang et al. 2018; Lu et al. 2018; Tambong 2017). The chp/tomA PAI encodes suites of putative serine proteases belonging to the Sbt, Chp, and Ppa families and carbohydrate activating enzymes (CAZymes) (Thapa et al. 2017). A few $C$. michiganensis effectors have been characterized, including the tomatinase tomA and the cellulase celA (Jahr et al. 2000; Meletzus et al. 1993). However, the plant targets of most effectors are unknown (Nandi et al. 2018).

Pioneering work in the late 1990s through the early 2010s resulted in vectors for gene deletion and expression in Clavibacter spp., but there is still reliance on these tools despite known limitations. Mutants depend on gene replacement with an antibiotic cassette or by transposon mutagenesis (Gartemann and Eichenlaub 2001; Kirchner et al. 2001; Peritore-Galve et al. 2021; Thapa et al. 2017). Engineering gene expression relies on using a plasmid modified from the backbone of native pCM1/pCM 2 plasmids from $C$. michiganensis. This requires recipient strains to lack the native plasmid, a confounding factor because known virulence 
genes are carried on pCM1/pCM2. Gene expression can also be achieved by transposition via a transposase-based vector, which can have unexpected effects depending on the genetic location inserted (Chalupowicz et al. 2012; Laine et al. 1996; Mullens and Jamann 2021; Tancos et al. 2013).

Genetic tool kits in other bacterial systems enabled functional characterization of genetic drivers in disease development. The original pSelAct vector was designed to make gene deletions in the foal actinobacterial pathogen, Rhodococcus equi (van der Geize et al. 2008). pSelAct was modified through the addition of a Gateway cassette into the multiple cloning site and was used to make single gene deletions in the distantly related actinobacterial plant pathogen Rhodococcus fascians (later referred to as pSelAct-KO [Savory et al. 2020]). A second group independently developed pMP201, for deletions in Streptomyces spp., which functions similarly to the pSelAct vector, using a positive selection marker and counter selection (Dubeau et al. 2008). Integrative expression vectors have been developed for gramnegative bacteria, but there are few examples in gram-positives. In Ralstonia solanacearum GMI1000 and related strains, the integrative plasmid series $\mathrm{pRC}$ enables insertion and expression of genetic material in a defined location (Monteiro et al. 2012). Finally, a knock-out vector using the sucrose counter-selection marker $s a c B$ was modified for integrative expression in Pseudomonas syringae pv. tomato DC3000 (Lee et al. 2018). Flexible genetic tools facilitate rapid advances in studying pathogens regardless of the system.

Here, we present a suite of genetic tools to facilitate investigation of Clavibacter spp., including a markerless deletion system, an integrative plasmid, and an $\mathrm{R}$ package for identification of permissive sites for plasmid integration. We optimized pSelAct-KO, a recombination-based system that allows for development of higherorder markerless deletions. pSelAct-KO was used to make a 5.6-kb deletion in the $C$. michiganensis chp/tomA PAI comprising five effectors ( $c h p E$ through $p p a C$ ), and the deletion was confirmed using whole-genome sequencing. We developed the R package permissR and pSelAct-Express to work in conjunction to allow for identification of permissive genomic locations and subsequent targeted plasmid integration. These genetic tools represent a clear advance in our ability to genetically investigate Clavibacter spp. by allowing for markerless deletions (decrease polar effects that occur from antibiotic insertions), enable the generation of higher-order mutants, and enable chromosomal expression (allows for gene expression and complementation without removal of endogenous plasmids). In total, these tools should accelerate reverse genetics and subsequent genotype-phenotype studies in Clavibacter spp.

\section{RESULTS AND DISCUSSION}

\section{pSelAct system for markerless deletions in Clavibacter spp.}

The utility of two-step systems has been demonstrated in other actinobacteria including Rhodococcus and Streptomyces bacteria as well as other gram-positive bacteria (Dubeau et al. 2008; Kostner et al. 2017; Savory et al. 2020). Thus, generating markerless deletions using selection and counter-selective methods could likely be applied Clavibacter and other related taxa. We employed the pSelAct-KO vector to $C$. michiganensis with the strategy illustrated in Figure 1 for construct design, selection, and counter-selection. In order to develop the knockout construct, approximately $1.5-\mathrm{kb}$ regions flanking the gene or gene locus of interest are amplified (Fig. 1A). Fragments from the $5^{\prime}$ and $3^{\prime}$ flanking regions are cloned into the pSelAct-KO Gateway C Cassette, through either Gateway cloning via LR Clonase from a Gateway pDONR vector or a homology-based cloning approach (InFusion or Gibson) to generate pSelAct $\Delta$ goi (Fig. 1B)
(Savory et al. 2020). pSelAct $\triangle$ goi is transformed into Escherichia coli, is screened via PCR, and is verified via Sanger sequencing, and is subsequently transformed into the wild-type Clavibacter strain (Fig. 1C). Positive recombinants are resistant to the antibiotic apramycin and are screened for their merodiploid state (haploid cells that have duplicated genetic material) using PCR (Fig. 1D). Growing the merodiploid on minimal medium supplemented with 5-fluorocytosine (5-FC) will promote a recombination event to generate a clean (scarless) deletion (Fig. 1E).

Determining apramycin resistance in C. michiganensis. The pSelAct-KO system employs selection using the aminoglycoside antibiotic apramycin to select for chromosomal integration, followed by counter-selection with 5-FC to recombine out. To determine the minimum inhibitory concentration (MIC) of apramycin, MIC growth curves were performed in C. michiganensis. In liquid rich tryptone broth with yeast (TBY) media, the MIC necessary to limit growth was $50 \mu \mathrm{g} / \mathrm{ml}$ (Fig. 2B). On solid agar TBY plates, the MIC required was higher at $100 \mu \mathrm{g} / \mathrm{ml}$ (Supplementary Fig. S1). Notably, if plates were incubated beyond 5 days post $-C$. michiganensis transformation, smaller colonies appeared to form (data not shown). This dissipated if Clavibacter transformants were plated on TBY that had double the MIC, at $200 \mu \mathrm{g} / \mathrm{ml}$. However, TBY plates with $100 \mu \mathrm{g} / \mathrm{ml}$ apramycin were sufficient for downstream screening (Supplementary Table S1). Susceptibility to the antibiotic apramycin in C. michiganensis differed on plates compared with other actinobacterial pathogens, which require lower concentrations (Savory et al. 2020). A TblastN search of the apramycinresistance gene in Clavibacter spp. (taxid: 1573) yielded no significant results and antibiotics within the same drug class, neomycin and gentamycin, are known to be effective (Supplementary Table S2) (Meletzus and Eichenlaub 1991). While it is unclear why a higher concentration is required, apramycin is effective against C. michiganensis.

Deletion of a 5.6-kb gene cluster in the tomato pathogen $\mathrm{C}$. michiganensis. To demonstrate the utility of the pSelAct-KO for gene deletion, we focused on the chp region in the PAI of C. michiganensis strain CASJ002. PAI-localized effectors have been of interest since their discovery and are hypothesized to drive disease development in the $C$. michiganensis-tomato pathosystem (Eichenlaub and Gartemann 2011; Nandi et al. 2018; Thapa et al. 2019). Within the $c h p E-p p a C$ region, there are several putative virulence genes, including four serine proteases and the virulence CAZyme pelAl (Fig. 2A). Three proteases are from the Ppa family and one from the Chp family. Four of the five genes of interest have been individually deleted (Chalupowicz et al. 2017; Thapa et al. 2017). Mutants of the proteases $c h p E$, ppaA, and ppaC had no reported effect on disease development when tested on tomato. Others have noted, however, that there may be functional redundancy and deleting one protease effector may not be sufficient for disease reduction (Peritore-Galve et al. 2021). The CAZyme pelAl mutant had reduced wilting symptoms on tomato. The protease ppaBl has not yet been tested for its contribution in disease development.

To test pSelAct-KO in $C$. michiganensis, we developed primers to amplify flanking regions before $\operatorname{ch} p E$ and after $p p a C$ (Fig. 2A). Using homology-based inFusion cloning, the fragments replaced the Gateway cassette of pSelAct-KO and a positive clone was selected and sequence-verified (Fig. 1A and B). Upon transformation of $C$. michiganensis wild-type strain CASJ002, the vector will recombine with one of the two homology arms (Fig. 1C). PCR was used to screen for the merodiploid state; first recombinants will provide two bands, one that is the same as wild type and one that is smaller, representative of the recombination event (Figs. 1D, a gray box highlights two 
amplicons, and $2 \mathrm{C}$ ). It is important to note that, as the deletion size increases, it may be more difficult to amplify the upper band representing the wild-type state. Since the merodiploid confers antibiotic resistance whereas the knockout does not, this phenotype can be used as a quick guide. However, if necessary, in the case of multigene deletions, it may be worthwhile to design a probe that binds to one of the flanking regions and to run a Southern blot.

To develop the knockout through secondary recombination, the merodiploid was plated in dilutions from $10^{-6}$ to $10^{-7}$ on minimal M9 (mM9) medium supplemented with the counterselectant 5-FC. Strains are required to be insensitive to the cysteine analog 5-FC but sensitive to its more toxic counterpart 5-florouracil (5-FU) and 5-fUMP (Dubeau et al. 2008). Multiple actinobacteria have been demonstrated to exhibit resistance to 5-FC at $100 \mu \mathrm{M}$, including Rhodococcus spp., Streptomyces spp., and C. sepedonicus (Dubeau et al. 2008; Savory et al. 2020; Syverson 2011; van der Geize et al. 2008). For counterselection, the gene cassette codA::upp converts 5-FC into the toxic compound 5-FU and subsequently 5-fUMP (van der Geize et al. 2008). After 7 to 14 days of growth, single colonies are visible. As a quick and easy screen, colonies from M9 5-FC plates were re-streaked on both TBY and apramycin-containing TBY plates. Recombination of the vector will inhibit growth on

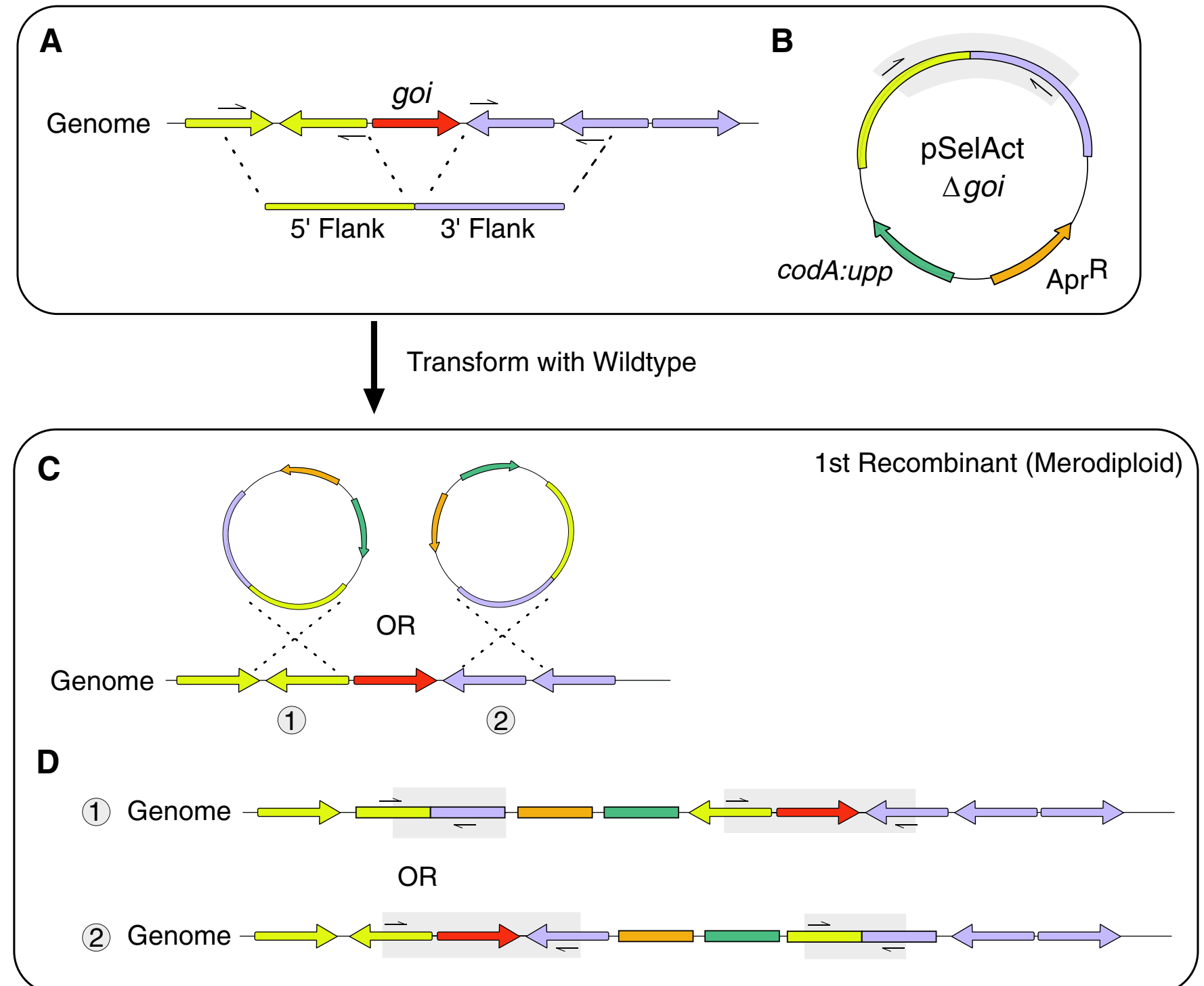

Counterselect on Minimal Media with 5-FC

$\mathbf{E}$

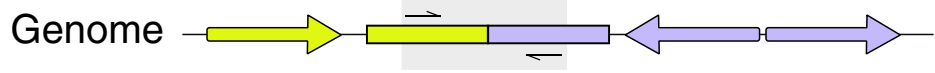

2nd Recombinant (Knockout)

Fig. 1. Pipeline for markerless knockouts using the vector pSelAct-KO. Gray-shaded area signifies the amplified region using primers spanning the region of interest. A, Amplification of flanking regions before and after genes of interest. B, Cloning of $5^{\prime}$ and $3^{\prime}$ flanking regions into pSelAct vector through either Gateway or homology-based cloning. C, Possible sites of recombination during transformation and selection of nonreplicating vector into the genome. D, Possible gene structure of merodiploid. E, Gene structure after recombination where gene or gene locus of interest is deleted. 
apramycin. Therefore, colonies growing on TBY but not TBY with apramycin either reverted back to wild type or are a true knockout (Fig. 1E). After incubation at $28^{\circ} \mathrm{C}$ on $\mathrm{M} 95-\mathrm{FC}$ plates, several hundred colonies from the $C$. michiganensis CASJ002 chpE-ppaC merodiploid were screened for second recombinants after transferring to apramycin-containing TBY plates. All colonies grew on both TBY and apramycincontaining TBY plates, indicating they remained merodiploids, likely showing ineffective compound uptake as no recombination events occurred.

We hypothesize that rapid growth could signal inefficient uptake of 5-FC leading to a nonselective environment. Therefore, adjustments to the incubation temperature were made. We grew the $C$. michiganensis CASJ002 chpE-ppaC merodiploid at $24^{\circ} \mathrm{C}$ on $\mathrm{M} 9$ 5-FC plates, which results in slower colony growth. Of the 63 colonies subsequently screened on both TBY and apramycin-containing TBY plates at $28^{\circ} \mathrm{C}$, three were confirmed to be true knockouts (Supplementary Fig. S2). Previous deletions using pSelAct or other similar systems tend to delete one to two genes at a time, so while we do not know the upper limits of the system, we were able to make a deletion as large as 5,636 bp in C. michiganensis CASJ002 (Fig. 2) (Savory et al. 2020). These results highlight the importance of environmental conditions impacting 5-FC uptake and effectiveness as means for counter-selection.

Admittedly, converting the merodiploid to a knockout mutant can be the most laborious and unpredictable step of the system. However, there are known approaches that can enhance recombination efficiency. First, the concentration of 5-FC could be optimized. We did not choose to alter this concentration, based on its success in distantly related actinobacteria and early reports of 5-FC resistance, 5-FU susceptibility in C. sepedonicus (Savory et al. 2020; Syverson 2011). Second, a similar system in Bacillus llicheniformis, pKVM4, noted that the addition of $\operatorname{cod} B$, an encoded cytosine permease, notably increased efficiency of selection (Kostner et al. 2017). Kostner et al. (2017) hypothesized that the addition of $\operatorname{cod} B$ increased 5-FC uptake, leading to an increase in the concentration of 5-FU and 5-fUMP present and, thus, greater selection. Re-engineering the pSelAct$\mathrm{KO}$ to include a cytosine permease homolog may improve

A

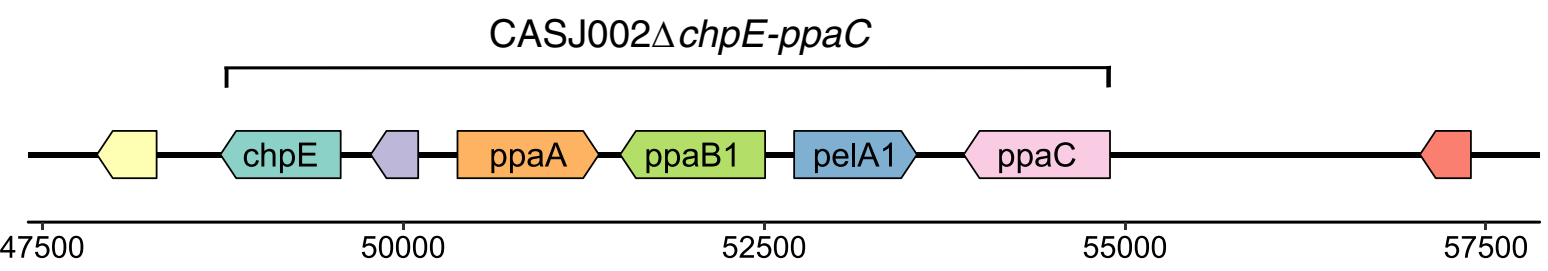

Position along Chromosome

B

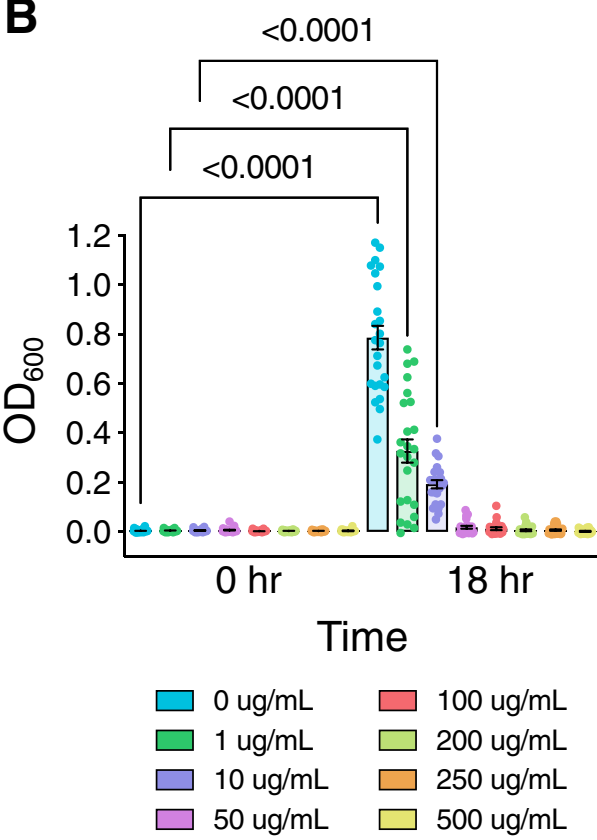

C

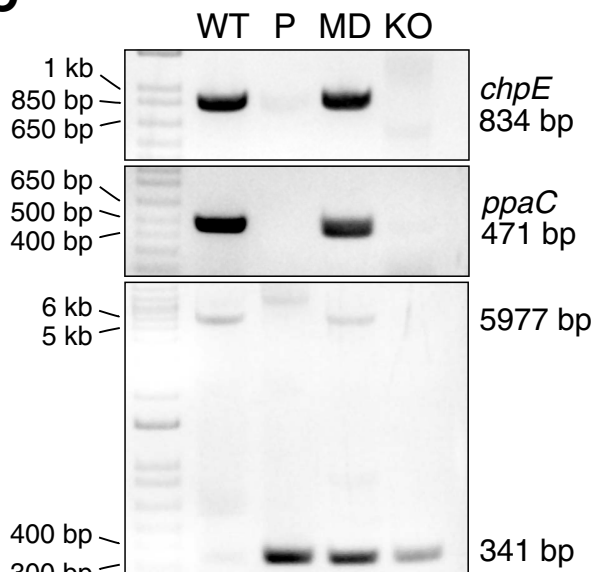

D

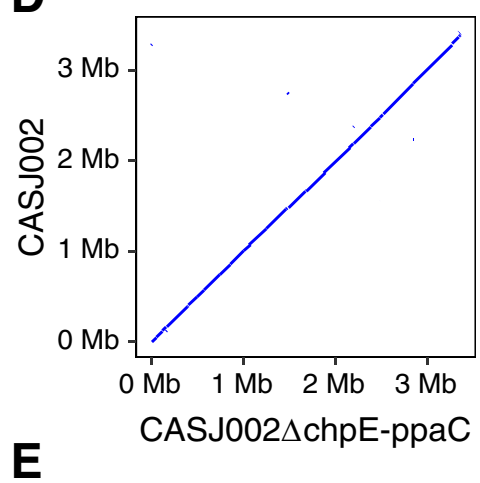

CASJ002

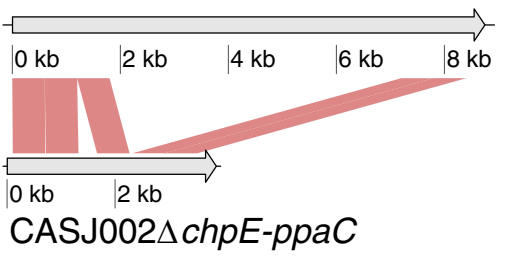

Fig. 2. Markerless deletion of chpE through ppaC in the tomato pathogen Clavibacter michiganensis CASJ002. A, Map of genes of interest for deletion and their flanking regions. B, Minimum inhibitory concentration of the antibiotic apramycin in tryptone broth with yeast liquid culture for $C$. michiganensis isolate CASJ002. Eight replicates per treatment were performed with media only as a negative control. The experiment was repeated independently three times. A one-way analysis of variance and a post-means Tukey's multiple comparisons test was computed. Comparisons of 0 - and 18 -h values from $50 \mu \mathrm{g} / \mathrm{ml}$ upward were not significantly different. C, PCR-based verification of deletion on 1\% Tris-acetate-EDTA agarose gel. The top panel, gene-specific primers for $c h p E$; the middle panel, gene specific primers for $p p a C$; and the bottom panel, primers that bind to the flanking regions and span the region deleted. Lanes are products from the reactions: WT $=$ wild type genomic DNA from CASJ002 (WT), P = plasmid DNA from Escherichia coli carrying the pSelAct_CASJ002_chpE-ppaC_KO, MD = genomic DNA from the merodiploid, amd KO = genomic DNA from CASJ002 $\Delta$ chpE-ppaC. The estimated sizes of the PCR products are depicted (bp = base pairs). D, Dot plot of CASJ002 $\Delta$ chpE-ppaC against reference CASJ002 genome sequence. E, Syntenic comparison of the region of interest from CASJ002 against the engineered deletion in CASJ002 $\Delta c h p E$-ppaC. Comparison fragment size is 600 bp. The gap in the $5^{\prime}$ flanking region of CASJ002 $\Delta$ chpE-ppaC represents a gap between the scaffolded contigs. 
recombination efficiency. Finally, modified environmental conditions can have dramatic effects. In the original pSelAct vector developed for $R$. equi, growing the merodiploid on rich medium over minimal medium demolished the counter-selective effects from 5-FC, likely due to a lack of 5-FC uptake related to the pyrimidine salvage pathway. While it is unclear whether decreasing the temperature had the same effect, it is clear that environmental conditions can impact recombination frequency.

Collectively, these results demonstrate that pSelAct-KO can be used in $C$. michiganensis to generate markerless deletions. Furthermore, optimizing the growth temperature when the bacteria are grown on M9 with 5-FC resulted in relatively high counter-selection. It is important to note that pSelAct-KO has been used in other gram-positive actinomycetes. Therefore, it is likely that this system will be functional in other Clavibacter species and related genera.

Sequence confirmation of $\mathrm{C}$. michiganensis CASJ002 $\Delta \mathrm{chpE}-$ $\mathrm{ppaC}$ reveal no major structural changes. The strong selection pressure from 5-FC compound conversion to 5-FU and 5-fUMP may result in plasmid loss or other structural changes. Plasmid loss, particularly pCM2, has been noted in past studies that genetically altered $C$. michiganensis (Stork et al. 2008). Of the three confirmed $C$. michiganensis CASJ002 $\Delta$ chpE-ppaC, only one lost the native plasmid pCM2 (Supplementary Fig. S2). In order to investigate for any potential large structural rearrangements, Illumina paired-end reads of $C$. michiganensis CASJ002 $\Delta$ chpE-ppaC colony 9 were assembled and compared with the wild-type genome (detailed methods below as well as in the GitHub repository pSelAct_KO_Clavibacter page). Even with low 13x Illumina sequencing coverage, most contigs were syntenic (Fig. 2D). A clean deletion in the region of interest was further confirmed between the wild type and deletion (Fig. 2E). Therefore, we recommend screening via PCR for one or more native plasmids as a background assessment and, if possible, low coverage whole-genome Illumina sequencing to compare against the wild-type genome as a precautionary measure.

Taken together, these data demonstrate the utility of using the pSelAct-KO system to generate markerless deletions in Clavibacter spp. This system has several advantages over antibiotic integration, including reduced potential for polar effects, and should facilitate generation of higher-order mutants. Since this system is markerless, higher-order deletions can be made, showcasing the potential strength of the system especially for characterizing redundant virulence genes or effectors.

\section{Prediction of permissive sites via permissR.}

In addition to establishing a system for markerless deletions in Clavibacter spp., we sought to establish an integrative plasmid for gene expression. Unlike previous integrative plasmids that were designed with a specific genome in mind, we wanted to design a system that was flexible for future genomic-driven functional studies. This required developing the necessary tools to predict potential permissive, noncoding target regions for plasmid integration.

We developed an $\mathrm{R}$ package, permiss $\mathrm{R}$, to predict permissive sites for potential plasmid integration using draft genomes. permissR minimally requires two input files, a GenBank file (.gbff or .gbk format) and a whole-genome FASTA file (Supplementary Fig. S3). We also recommend running ISEScan to avoid cloning and recombining into an insertion element that could cause unwanted structural changes. Insertion element prediction can be run within the $\mathrm{R}$ console or separately on the terminal and imported into permissR. Upon running permissR, either by providing paths to the required files or using the pop-up graphical use interface (GUI) to select the files, the pipeline will provide several outputs. Outputs include a whole-genome plot with information on the annotation, insertion elements (if present), and a scanning 1,000-bp window of Shannon entropy and GC content, two different measurements of complexity (Fig. 3A) (Akhter et al. 2013). Predicted candidate permissive sites (otherwise known as intergenic regions) are at least $1.5 \mathrm{~kb}$ in size, are not within insertion elements, and are located on contigs a minimum $15 \mathrm{~kb}$ in size. While sites for homologous recombination can be smaller in size (i.e., $1 \mathrm{~kb}$ ), since 1.5 -kb regions have been reliable in terms of recombination efficiency and specificity and other studies using similar approaches selected similar sizes, we opted to maintain candidate sites be a minimum $1.5 \mathrm{~kb}$ in size (Dubeau et al. 2008; Monteiro et al. 2012; Savory et al. 2020). Plots of candidate sites are output such that the user can refer to the neighboring genes (Fig. 4B). Predicted sites are ranked and supplied to the user as a tab-delimited text file. Additionally, candidate sites are also compared against the whole genome and, if close hits are present (i.e., the maximum number of mismatches equal $15 \%$ or less difference based on permissive hit length), they are exported in a separate tabdelimited text file. Permissive regions predicted by permissR are not assessed or filtered in the context of lethality or having a potential defect to fitness. Therefore, users interested in permissR should test several regions for effects on bacterial fitness, especially if the genome has not been used with permissR before.

Bacterial assembly and annotation quality should be independently assessed before running permissR. Since the pipeline relies on space between coding information, permissR will not predict sites on bacterial genomes that are too fragmented. Contigs less than the $15-\mathrm{kb}$ minimum size will not be assessed. While permissR itself does not assess annotation quality, results are dependent on a reliable annotation. Common measurements of annotation quality include BUSCO scores, the number of coding genes, and the number of hypothetical proteins. If the BUSCO scores are low or if the number of coding genes or hypothetical proteins becomes unreasonable compared with related taxa, then the genome should be improved before proceeding (Richardson and Watson 2013).

To test permissR, we used the $C$. michiganensis CASJ002 strain to predict potential sites. permissR predicted 10 candidate sites in the CASJ002 genome (Fig. 3A; Supplementary Table $\mathrm{S} 3$ ). Of the 10 candidates, one had a homologous region within the genome, which happened to be the second top predicted site (Fig. 3B). The GC content of sites ranged from 55 to $75 \%$. Additional Clavibacter genomes were tested against the pipeline to see if sites could be detected in other species (Supplementary Table S4). The number of predicted sites differed across Clavibacter strains and species, including bacteria outside the Clavibacter genus (Supplementary Table S4). This pipeline could, in theory, be used to help other researchers design integrated plasmids for their system of interest as permissR is not genera dependent.

\section{Green fluorescent protein (GFP) expression in the Clavibacter chromosome, using pSelAct Express.}

Next, we sought to experimentally validate two candidate sites identified by permissR. First, we generated an integrative plasmid, pSelAct-Express containing GFP. pSelAct-Express was built from the pSelAct-KO vector by replacing the $\operatorname{codA}::$ upp counter-selection cassette with the expression cassette from pKGT-GFP, which includes the phage promoter pCMP1 and enhanced GFP (eGFP) (Fig. 4A) (Chalupowicz et al. 2012; Tancos et al. 2013). We developed primers with homologous ends to amplify the pSelAct fragment and pCMP1::eGFP casette and cloned them together, using inFusion. Similar to pSelAct-KO, to use pSelAct-Express, the Gateway cassette is replaced with an approximately $1.5-\mathrm{kb}$ region amplified from within one of the predicted permissive sites to facilitate recombination. 
For testing the system in $C$. michiganensis CASJ002, approximately $1.5-\mathrm{kb}$ regions within two permissive regions, hit regions 1 and 3 (HR1 and HR3), were cloned into pSelAct-Express (Fig. 4B; Supplementary Table S5). Each construct was separately transformed into CASJ002 and construct integration was verified by PCR (Fig. 4B and C). Expression of eGFP was confirmed by confocal microscopy, using wild-type CASJ002 as a negative control (Fig. 4D). To verify plasmid integration did not affect disease development and bacterial titers, 3-week-old tomato cultivar M82 plants were stab-inoculated. There were no significant differences between wild type and the integrated variants when measuring the area of stem canker, a signature symptom in C. michiganensis disease development in tomato (Fig. 4E and F). Wilting, another common disease phenotype from $C$. michiganensis-tomato infections, was not explicitly measured; however, no drastic changes in wilting were observed. Similarly, no significant differences were detected in bacterial titer $1 \mathrm{~cm}$ above the site of inoculation (Fig. 4G). While we did not observe alterations in bacterial fitness or disease development upon plasmid integration in HR1 or HR3, we recommend testing several candidate regions, as there is always a potential for unpredictable effects. These results demonstrate permissR can be used to discover promising sites for plasmid integration using pSelAct-Express. We were able to identify two chromosomal regions for integrative expression in $C$. michiganensis that do not affect bacterial virulence.

\section{Conclusion.}

The tools optimized and developed in this work, pSelAct-KO, permissR, and pSelAct-Express, provide flexibility and some degree of throughput for genetic manipulation of Clavibacter bacteria. Molecular tools that enable the easier manipulation for genotype-phenotype studies have greatly advanced our understanding of these organisms, and we conclude that the tools

A

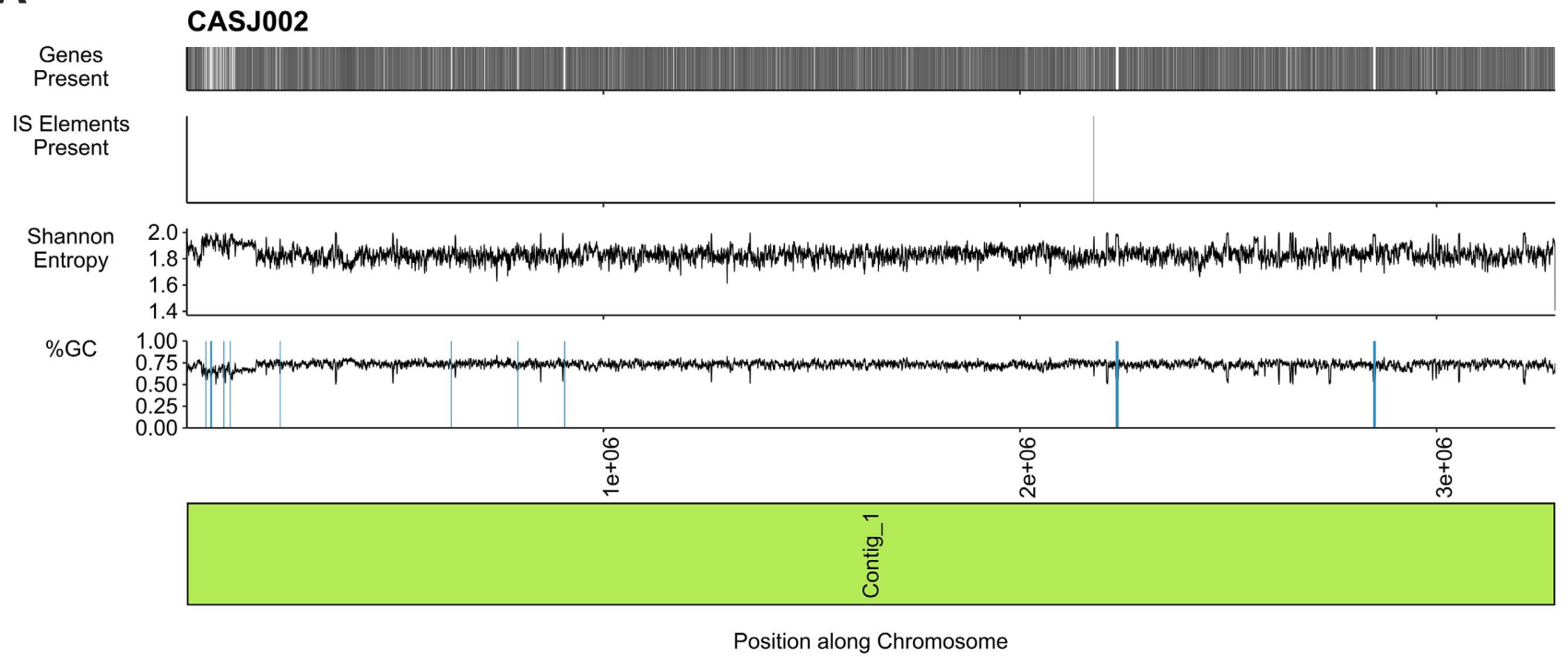

B
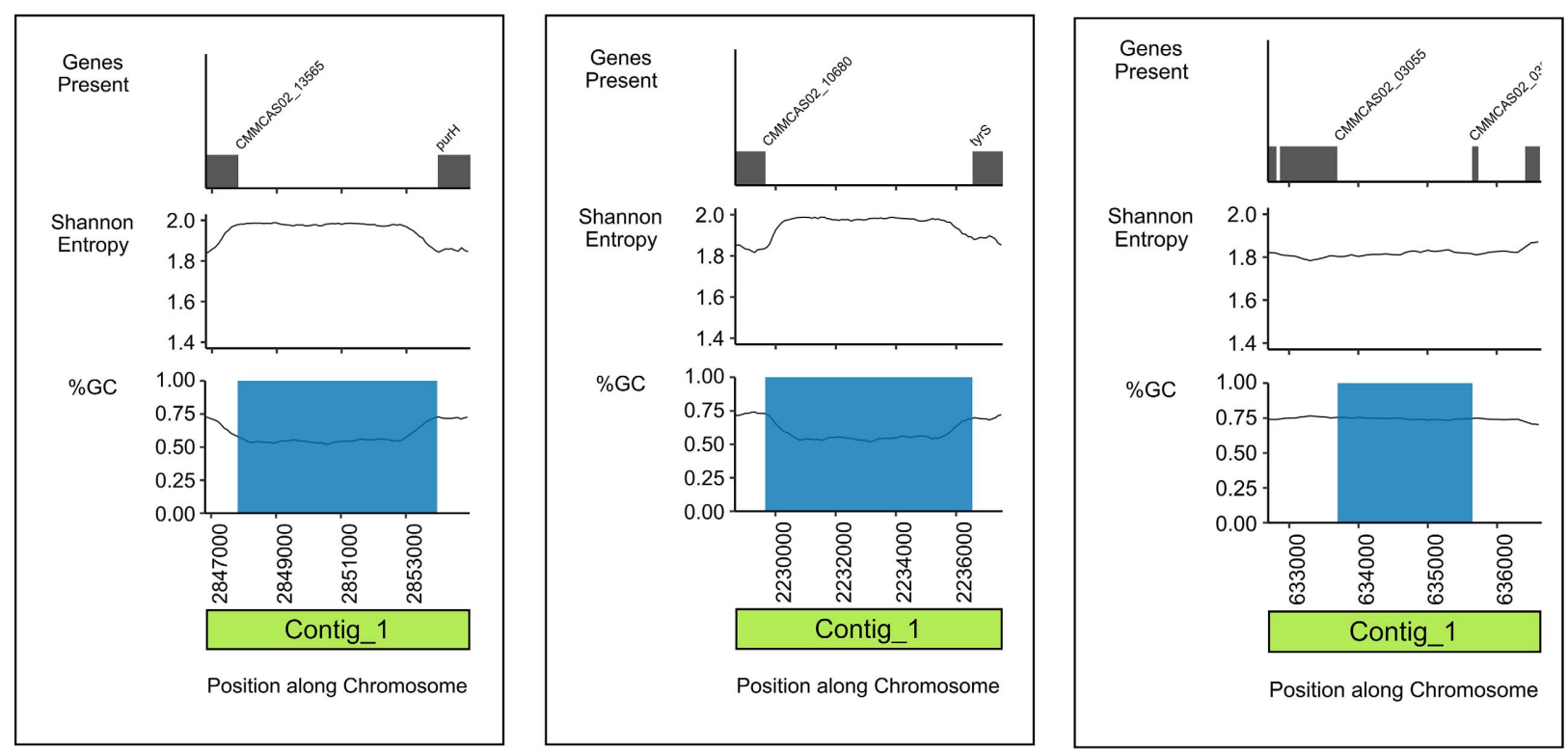

Fig. 3. The R package permissR detects permissive sites in the tomato pathogen Clavibacter michiganensis CASJ002. A, Whole-genome analyses to detect permissive sites. From top to bottom, respectively, the presence and absence of genes encoded, the functional insertion elements (IS element) predicted in the genome by ISEScan, a 1,000-bp scanning window calculation of Shannon entropy, and a 1,000-bp scanning window of GC content calculated along the genome. Candidate permissive sites highlighted in blue. B, Subplots of predicted permissive sites ranked by GC content and Shannon entropy. Left to right, the top two ranked sites and the last ranked site. 


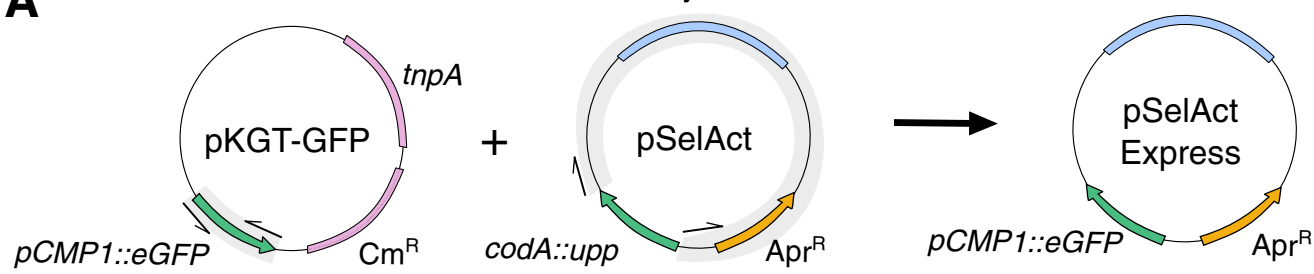

B

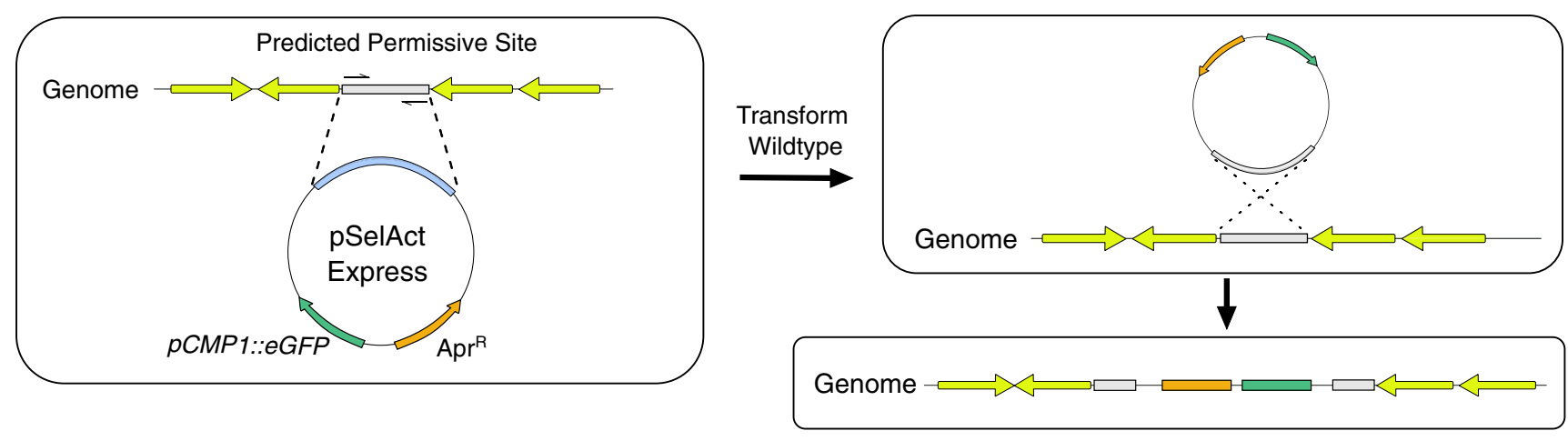

C

D

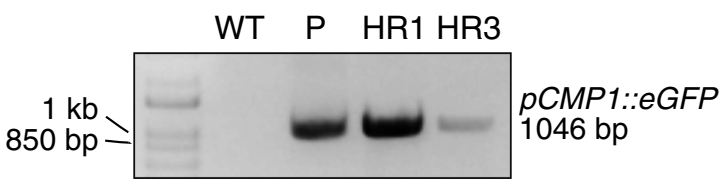

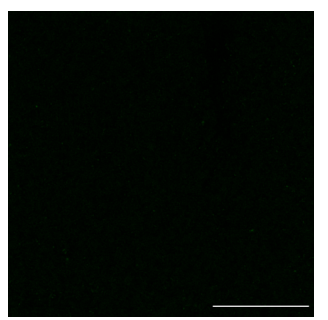

WT

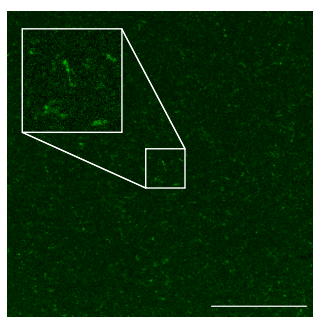

HR1

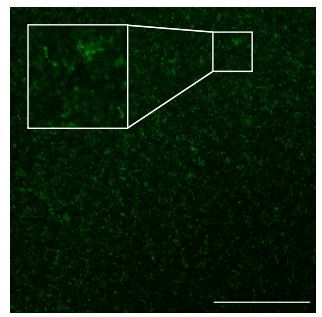

HR3

\section{E}
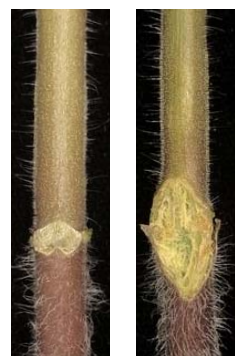

Mock

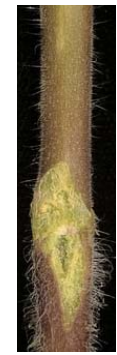

HR1
F

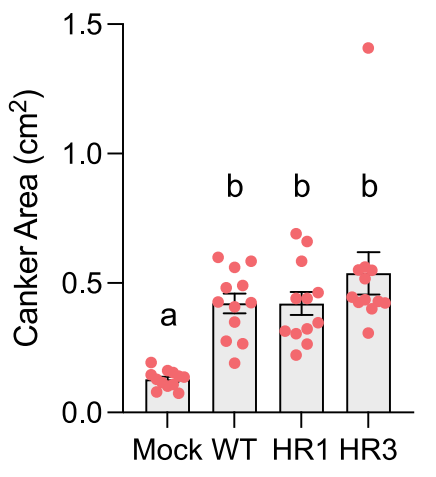

Treatment
G

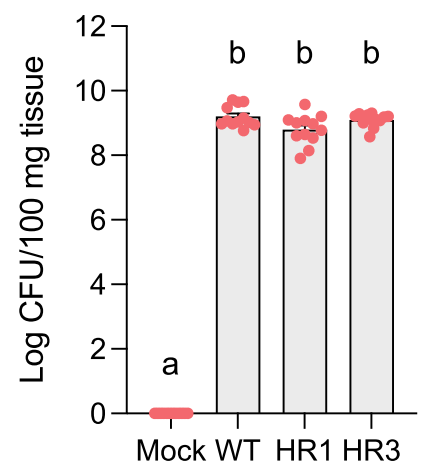

Treatment

Fig. 4. Enhanced green fluorescent protein (eGFP) expression in two Clavibacter permissR target regions using an integrative plasmid. A, pSelAct Express was developed by replacing the codA::upp counter-selection cassette in pSelAct-KO with eGFP under a phage promoter from pKGT-GFP. B, On the left, amplification of a predicted permissive site from permissR into pSelAct-Express via Gateway or homology-based cloning. On the right, transformation with the modified nonreplicating vector into the wild-type (WT) strain causes recombination into the genome at the predicted permissive site. C, PCR-based verification of the plasmid insertion via amplification of $p C M P 1:: e G F P$. WT = genomic DNA from CASJ002, P = plasmid DNA from Escherichia coli carrying the pSelAct Express, HR1 = genomic DNA from CASJ002 integration variant at hit region 1, HR3 = genomic DNA from CASJ002 integration variant at hit region 3. D, Confocal microcopy of eGFP expression in C. michiganensis CASJ002 (WT) and transformed integration variants (HR1 and HR3). White bar represents $40 \mu \mathrm{m}$ in length. E, Representative tomato canker symptoms after inoculation with mock (water), WT CASJ002, and integrative variants HR1 and HR3. Stems were stabbed with a needle and were inoculated with $5 \mu$ l of inoculum (either water or bacteria at optical density at $600 \mathrm{~nm}=1$ ). Images were taken at 14 days postinoculation (dpi). Four plants per treatment per experiment were conducted with the entire experiment repeated three independent times. F, Quantification of canker area shown in E. Measurements were normalized with a 1-cm ruler (not shown). A one-way analysis of variance (ANOVA) and a post-means Tukey's multiple comparisons test were computed. G, Bacterial titers in stem tissue $14 \mathrm{dpi}$ at $1 \mathrm{~cm}$ above the site of inoculation from tomatoes shown in E and F. A one-way ANOVA and a post-means Tukey's multiple comparisons test were computed. 
presented here will open the door to investigating Clavibacter spp. Designed with flexibility in mind, we think there is a potential to be adapted to other orphan systems beyond bacteria in the Clavibacter genus.

\section{MATERIALS AND METHODS}

\section{Plasmids, stains, and culture conditions.}

Clavibacter michiganensis strains were grown in tryptone broth with yeast (TBY) (Kirchner et al. 2001). Clavibacter transformants were selected on TBY rich media supplemented with $200 \mu \mathrm{g}$ of apramycin per milliliter and were screened on TBY with only $100 \mu \mathrm{g}$ of apramycin per milliliter (Alfa Aesar, Haverhill, MA, U.S.A.). Escherichia coli was grown in Luria broth at $37^{\circ} \mathrm{C}$. All strains were grown under standard temperatures, as listed in Supplementary Table S1, except when selecting for second recombinants. This process requires growth on a minimal media, M9, supplemented with $100 \mu \mathrm{g}$ of of 5-FC and grown at $24^{\circ} \mathrm{C}$, as listed in Supplementary Table S1 (Stork et al. 2008) (VWR, Radnor, PA, U.S.A.). A $10-\mathrm{mg} / \mathrm{ml}$ stock solution of 5-FC was prepared in distilled water and was filter-sterilized. Apramycin was prepared similarly, at a stock concentration of $100 \mathrm{mg} / \mathrm{ml}$.

\section{MIC of apramycin in Clavibacter spp.}

Initial starter cultures were grown overnight at $28^{\circ} \mathrm{C}$ with shaking at $200 \mathrm{rpm}$ in $5 \mathrm{ml}$ of TBY media. Cultures were spun down at max speed for 2 min and were washed with and resuspended in sterilized water. The optical density at $600 \mathrm{~nm}\left(\mathrm{OD}_{600}\right)$ was taken and was adjusted to a final concentration of 0.01 in TBY with different antibiotic concentrations. Eight 200- $\mu$ l wells were used for each antibiotic concentration in a clear 96-well plate (Beckman Coulter, Pasadena, CA, U.S.A.) and were incubated at $28^{\circ} \mathrm{C}$ with shaking at $200 \mathrm{rpm}$. $\mathrm{OD}_{600}$ measurements were taken at 0 and $18 \mathrm{~h}$ Experiments were repeated a minimum three times.

\section{pSelAct-KO and pSelAct-Express.}

For generation of the multigene deletion plasmid, genomic DNA was extracted from 5-ml cultures of $C$. michiganensis CASJ002 grown overnight at $28^{\circ} \mathrm{C}$ with shaking at $200 \mathrm{rpm}$, using a similar protocol of extraction (Murray and Thompson 1980). Approximately $1.5-\mathrm{kb}$ regions flanking clustered genes of interest were amplified using PCR. It is important to select the position of the knockout carefully to avoid deletion into nearby protein-coding genes to prevent unwanted polar effects. The reaction mixture for PCRs was as follows: $1 \times$ iProof GC buffer, $0.2 \mu \mathrm{M}$ of each primer, 50 to $200 \mathrm{ng}$ of genomic DNA, 2\% dimethyl sulfoxide, $0.2 \mu \mathrm{M}$ of DNTPs, and double distilled water to a final volume of $20 \mu \mathrm{l}$. Extension times were 15 to $30 \mathrm{~s}$ per kilobase of DNA amplified. Primer sequences, amplicon size, and optimized annealing temperatures for each primer pair are listed in Supplementary Table S5. The backbone of pSelAct was linearized using Phusion polymerase with GC-buffer, requiring no more than $15 \mathrm{ng} / \mu \mathrm{l}$. Amplicons were gel-purified using the Zymo gel extraction kit according to the manufacturer instructions (Irvine, CA, U.S.A.). The knockout construct was developed through inFusion, using the purified amplicons, based on the manufacturer instructions, with the insert to vector ratio of 2:1 (Takara Bio, Mountain View, CA, U.S.A.) and transformed into E. coli DH5 $\alpha$. The resulting plasmid insertions were amplified and confirmed via Sanger sequencing.

For generation of pSelAct Express, pKGT-GFP plasmid DNA was extracted from E. coli, using a QIAprep Spin miniprep kit, according to manufacturer instructions (Qiagen, Hilden,
Germany). The phage promoter eGFP expression cassette ( $p C M P 1:: e G F P$ ) was amplified via PCR (Supplementary Table S5). The pSelAct plasmid was linearized without codA::upp, using conditions similar to those stated above. Similar to generating the knockout construct, the plasmid was generated using inFusion, was transformed into $E$. coli $\mathrm{DH} 5 \alpha$, was screened via $\mathrm{PCR}$, and was confirmed via Sanger Sequencing.

For cloning into hit regions from permissR predictions in $C$. michiganensis CASJ002, primers were designed from output sequence to amplify regions approximately 1.4 to $1.5 \mathrm{~kb}$ in size. HR1 and HR3 were amplified from genomic DNA and were cloned to replace the Gateway cassette via InFusion (Supplementary Tables S3 and S5). Positive clones were confirmed as above.

\section{Transformation and selection.}

Clavibacter competent cells were prepared and transformed as previously described (Kirchner et al. 2001). Briefly, cells were transformed with 100 to $200 \mathrm{ng}$ of plasmid DNA and were plated on TBY with $200 \mu \mathrm{g}$ of apramycin per milliliter. Colonies were picked and re-streaked onto TBY with $100 \mu \mathrm{g}$ of apramycin per milliliter. Colonies were cultured overnight in TBY with $100 \mu \mathrm{g}$ of apramycin per milliliter, for genomic DNA extraction (Murray and Thompson 1980).

\section{Selection for second recombinant.}

Positive merodiploids were grown overnight at their respective temperature and media. The culture was centrifuged at max speed, and the pellet resuspended in $1 \mathrm{ml}$ of sterile water. Tenfold dilutions from $10^{-1}$ to $10^{-7}$ were made and $100 \mu \mathrm{l}$ from $10^{-6}$ and $10^{-7}$ dilutions were plated onto $\mathrm{mM} 9$ medium supplemented with $100 \mu \mathrm{g}$ per milliliter of 5-FC. Plates were incubated at their indicative lower temperature, $24^{\circ} \mathrm{C}$ and were optimized for counter-selection (Supplementary Table S1) for 9 to 14 days. To screen quickly for recombination events, colonies were replated onto their indicated rich media with and without apramycin selection. Positive colonies, which only grew on the rich media, were additionally screened via PCR using both span and gene-specific primers, for a true knockout and not a recombinant that has returned to wild-type state.

\section{Bioinformatic analyses.}

C. michiganensis CASJ002 $\Delta$ chpE-ppaC genomic DNA was prepared from 5-ml overnight cultures (Murray and Thompson 1980). The Microbial Genome Sequencing Center (MIGS) library prepped the DNA for paired-end read sequencing via an Illumina platform. Raw paired-end reads were checked for quality and any contamination using FastQC (v0.11.9) and the sendsketch.sh script from bbtools, respectively. Since contamination was present, genomes closest to the top one to two hits from the sendsketch.sh output were downloaded using the bioinf_tools package (v1.8.17) (Lee 2018). Reads were then mapped to the genome of the Clavibacter reference and contaminants and were binned to their respective hit using bbtools' bbsplit.sh script. Successful binning was confirmed using sourmash (v3.5.0) based on a kmer size of 31 (Brown and Irber 2016). Merged reads were reformed via reformate.sh from bbtools and were trimmed using trimmomatic (v0.39). Trimmed reads were de novo assembled via SPAdes (v3.14.1) with default parameters for short-read assembly (Bankevich et al. 2012). Contigs were scaffolded using MeDuSa based on the reference wildtype genome and were oriented based on the reference, using CONTIGuator2 (Bosi et al. 2015; Galardini et al. 2011). The scaffolded contigs and the region of interest were mapped to their associate reference via Minimap2 (v2.17-r941) and FastANI (v1.32.0), and structural changes were assessed using a custom $\mathrm{R}$ script that uses the $\mathrm{R}$ package pafr $(0.0 .2)$ and genoplotR (0.8.11) (Charif and Lobry 2007; Jain et al. 2018; 
Li 2018). Detailed methods and scripts can be found in the GitHub repository.

\section{The $R$ package permiss $R$.}

permissR was written in the $R$ language ( $R$ Core Team 2019) and requires only two input files, at minimum, a GenBank file (gbk or gbff file formate) and a FASTA file. While the package does not require any external programs to run, it is recommended running and including the output from ISEScan, an annotation-independent software to find insertion elements in bacterial genomes, to avoid the potential of cloning into a functional insertion element that may cause unwanted gene movement (Xie and Tang 2017). To run ISEScan, we recommend using the package management software bioconda.

Detailed installation and usage can be found in the permissR GitHub repository. Briefly, installation of the package requires installing the devtools $\mathrm{R}$ package. To run, calling the function permiss $\mathrm{R}$ in the package on the $\mathrm{R}$ console, the user can provide the path (relative or absolute) for the GenBank file and then FASTA file of interest or leave the function empty, which will cause a GUI to appear to allow the user to select each file. The function will ask the name of the strain, which is used to label the output files, as well as if there are any outputs for ISEScan.

\section{Plant growth and pathogenicity assays.}

The tomato cultivar M82 (Solanum lycopersicum cv. M82) was used for all assays. Tomato plants were grown in a growth chamber under 16-h light and 8-h dark conditions with 50\% humidity. Plants were grown for 3 weeks, by which time at least two true leaves had fully emerged, were pricked between the two cotyledons on the stem using a sterilized needle, and $5 \mu \mathrm{l}$ of bacterial inoculum or water (mock) was dropped into the wound. The inoculum was prepared by initially streaking out Clavibacter stocks 4 days before inoculation on either TBY or TBY plates with apramycin. The day of inoculation, bacteria were suspended from the plate in sterile water and the $\mathrm{OD}_{600}$ was adjusted to 1 . Symptoms were photographed at 14 days postinoculation (dpi) and the canker was measured, using ImageJ, based on a $1-\mathrm{cm}$ ruler to normalize.

To determine bacterial titers in the tomato stem, stem segments were cut surrounding the inoculation site at $14 \mathrm{dpi}$. Stem pieces were surface-sterilized in $70 \%$ ethanol for 10 to $15 \mathrm{~s}$, followed by suspension in sterile water for 10 to $15 \mathrm{~s}$. Sections were taken $1 \mathrm{~cm}$ above the inoculation site. Tissue was weighed at $100 \mathrm{mg}$, was suspended in sterile water, and was ground. Sterile dilutions of the ground tissue were plated on D2 medium supplemented with $20 \mathrm{mg}$ of cycloheximide and were incubated for 5 to 6 days at $28^{\circ} \mathrm{C}$ (Thapa et al. 2017).

\section{Confocal microscopy.}

Before imaging, Clavibacter stocks were streaked onto either TBY or TBY plates with apramycin and were grown at $28^{\circ} \mathrm{C}$. Bacteria was smeared onto glass slides, $2 \mu \mathrm{l}$ of sterile water was pipetted on, and glass cover slips were added on top. Confocal images were taken with a Leica SP8 TCS with a $63 \times$ oil objective, laser power set to $6 \%$, and excitation and emission wavelengths set to 488 and $509 \mathrm{~nm}$, respectively. Fiji with the Bio-Formats Importer was used to adjust the brightness and contrast of the images.

\section{Statistical analyses.}

All raw data were plotted and error bars in plots represent standard error of mean. Data across independent replicates was colligated and a one-way analyis of variance and a post-means Tukey's multiple comparisons test was computed, using Graphpad Prism 9 software. Significant differences between groups include $P$ values $\leq 0.001$. Outputs of statistical analyses from
Figures $2 \mathrm{~B}$ and $4 \mathrm{~F}$ and $4 \mathrm{G}$ can be found in Supplementary Tables S6, S7, and S8, respectively.

\section{Data availability.}

Paired-end Illumina reads of $C$. michiganensis CASJ002 $\Delta c h p E$ ppaC were submitted to figshare and the assembled genome is available in the GitHub repository, along with the scripts necessary for analyzing the data. The $\mathrm{R}$ package permissR can also be found in the GitHub repository, including installation and usage instructions.

\section{ACKNOWLEDGMENTS}

We thank J. Chang from Oregon State University for providing the pSelAct vector and Qingyang Lyu for Sanger sequencing it. We also thank MIGS for Illumina sequencing as well as N. Carleson and Z. Foster for the suggestion of turning permissR into an $\mathrm{R}$ package. Finally, we thank the members of the Coaker lab for their thoughtful discussion and reading of the manuscript.

\section{AUTHOR-RECOMMENDED INTERNET RESOURCES}

figshare Illumina reads:

https://figshare.com/articles/dataset/CASJ002_chpE-ppaC_Paired_ End_Illumina_Raw_Reads/14810322

GitHub repository, assembled Clavibacter genome:

https://github.com/DanielleMStevens/pSelAct_KO_Clavibacter

GitHub repository, permissR:

https://github.com/DanielleMStevens/permissR

\section{LITERATURE CITED}

Ahmad, A., Mbofung, G. Y., Acharya, J., Schmidt, C. L., and Robertson, A. E. 2015. Characterization and comparison of Clavibacter michiganensis subsp. nebraskensis strains recovered from epiphytic and symptomatic infections of maize in Iowa. PLoS One 10:e0143553.

Akhter, S., Bailey, B. A., Salamon, P., Aziz, R. K., and Edwards, R. A. 2013. Applying Shannon's information theory to bacterial and phage genomes and metagenomes. Sci. Rep. 3:1033.

Bankevich, A., Nurk, S., Antipov, D., Gurevich, A. A., Dvorkin, M., Kulikov, A. S., Lesin, V. M., Nikolenko, S. I., Pham, S., Prjibelski, A. D., Pyshkin, A. V., Sirotkin, A. V., Vyahhi, N., Tesler, G., Alekseyev, M. A., and Pevzner, P. A. 2012. SPAdes: A new genome assembly algorithm and its applications to single-cell sequencing. J. Comput. Biol. 19:455-477.

Bentley, S. D., Corton, C., Brown, S. E., Barron, A., Clark, L., Doggett, J., Harris, B., Ormond, D., Quail, M. A., May, G., Francis, D., Knudson, D., Parkhill, J., and Ishimaru, C. A. 2008. Genome of the actinomycete plant pathogen Clavibacter michiganensis subsp. sepedonicus suggests recent niche adaptation. J. Bacteriol. 190:2150-2160.

Bosi, E., Donati, B., Galardini, M., Brunetti, S., Sagot, M.-F., Lió, P. Crescenzi, P., Fani, R., and Fondi, M. 2015. MeDuSa: A multi-draft based scaffolder. Bioinformatics 31:2443-2451.

Brown, C. T., and Irber, L. 2016. sourmash: A library for MinHash sketching of DNA. J. Open Source Softw. 1:27.

Chalupowicz, L., Barash, I., Reuven, M., Dror, O., Sharabani, G. Gartemann, K., Eichenlaub, R., Sessa, G., and Manulis-Sasson, S 2017. Differential contribution of Clavibacter michiganensis ssp. michiganensis virulence factors to systemic and local infection in tomato. Mol. Plant Pathol. 18:336-346.

Chalupowicz, L., Zellermann, E.-M., Fluegel, M., Dror, O., Eichenlaub, R., Gartemann, K.-H., Savidor, A., Sessa, G., Iraki, N., Barash, I., and Manulis-Sasson, S. 2012. Colonization and movement of GFP-labeled Clavibacter michiganensis subsp. michiganensis during tomato infection. Phytopathology 102:23-31.

Charif, D., and Lobry, J. R. 2007. SeqinR 1.0-2: A contributed package to the $\mathrm{R}$ project for statistical computing devoted to biological sequences retrieval and analysis. Pages 207-232 in: Structural Approaches to Sequence Evolution. U. Bastiola, M. Porto, E. Roman, and M. Vendruscolo, eds. Springer-Verlag, Berlin.

Dalio, R. J. D., Herlihy, J., Oliveira, T. S., McDowell, J. M., and Machado, M. 2018. Effector biology in focus: A primer for computational prediction and functional characterization. Mol. Plant-Microbe Interact. 31:22-33.

Dubeau, M.-P., Ghinet, M. G., Jacques, P.-É., Clermont, N., Beaulieu, C., and Brzezinski, R. 2008. Cytosine deaminase as a negative selection 
marker for gene disruption and replacement in the genus Streptomyces and other actinobacteria. Appl. Environ. Microbiol. 75:1211-1214.

Eichenlaub, R., and Gartemann, K.-H. 2011. The Clavibacter michiganensis subspecies: Molecular investigation of gram-positive bacterial plant pathogens. Annu. Rev. Phytopathol. 49:445-464.

Galardini, M., Biondi, E. G., Bazzicalupo, M., and Mengoni, A. 2011. CONTIGuator: A bacterial genomes finishing tool for structural insights on draft genomes. Source Code Biol. Med. 6:11.

Gartemann, K.-H., and Eichenlaub, R. 2001. Isolation and characterization of IS1409, an insertion element of 4-chlorobenzoate-degrading Arthrobacter sp. strain TM1, and development of a system for transposon mutagenesis. J. Bacteriol. 183:3729-3736.

Hwang, I. S., Oh, E., Kim, D., and Oh, C. 2018. Multiple plasmid-borne virulence genes of Clavibacter michiganensis ssp. capsici critical for disease development in pepper. New Phytol. 217:1177-1189.

Jahr, H., Dreier, J., Meletzus, D., Bahro, R., and Eichenlaub, R. 2000. The endo- $\beta$-1,4-glucanase CelA of Clavibacter michiganensis subsp. michiganensis is a pathogenicity determinant required for induction of bacterial wilt of tomato. Mol. Plant-Microbe Interact.. 13:703-714.

Jain, C., Rodriguez-R, L. M., Phillippy, A. M., Konstantinidis, K. T., and Aluru, S. 2018. High throughput ANI analysis of $90 \mathrm{~K}$ prokaryotic genomes reveals clear species boundaries. Nat. Commun. 9:5114.

Kirchner, O., Gartemann, K.-H., Zellermann, E.-M., Eichenlaub, R., and Burger, A. 2001. A highly efficient transposon mutagenesis system for the tomato pathogen Clavibacter michiganensis subsp. michiganensis. Mol. Plant-Microbe Interact.. 14:1312-1318.

Kostner, D., Rachinger, M., Liebl, W., and Ehrenreich, A. 2017. Markerless deletion of putative alanine dehydrogenase genes in Bacillus licheniformis using a codBA-based counterselection technique. Microbiology 163:1532-1539.

Laine, M. J., Nakhei, H., Dreier, J., Lehtilä, K., Meletzus, D., Eichenlaub, R., and Metzler, M. C. 1996. Stable transformation of the grampositive phytopathogenic bacterium Clavibacter michiganensis subsp. sepedonicus with several cloning vectors. Appl. Environ. Microbiol. 62:1500-1506.

Lee, M. D. 2018. Bioinformatics tools (bit). Zenodo. Geneva, Switzerland. Published online.

Lee, Y.-C., Chien, C.-F., and Lin, N.-C. 2018. Knock-out or knock-in? Converting a SacB-based gene disruption system for site-specific chromosomal integration in Pseudomonas syringae pv. tomato DC3000. J. Microbiol. Methods 145:50-58.

Li, H. 2018. Minimap2: Pairwise alignment for nucleotide sequences Bioinformatics 34:3094-3100.

Lu, Y., Ishimaru, C. A., Glazebrook, J., and Samac, D. A. 2018. Comparative genomic analyses of Clavibacter michiganensis subsp. insidiosus and pathogenicity on Medicago truncatula. Phytopathology 108:172-185.

Meletzus, D., Bermphol, A., Dreier, J., and Eichenlaub, R. 1993. Evidence for plasmid-encoded virulence factors in the phytopathogenic bacterium Clavibacter michiganensis subsp. michiganensis NCPPB382. J. Bacteriol. 175:2131-2136.

Meletzus, D., and Eichenlaub, R. 1991. Transformation of the phytopathogenic bacterium Clavibacter michiganense subsp. michiganense by electroporation and development of a cloning vector. J. Bacteriol. 173: 184-190.

Monteiro, F., Solé, M., van Dijk, I., and Valls, M. 2012. A chromosomal insertion toolbox for promoter probing, mutant complementation, and pathogenicity studies in Ralstonia solanacearum. Mol. Plant-Microbe Interact. 25:557-568.

Mullens, A., and Jamann, T. 2021. Colonization and movement of green fluorescent protein-labeled Clavibacter nebraskensis in maize. Plant Dis. 105:1422-1431.

Murray, M. G., and Thompson, W. F. 1980. Rapid isolation of high molecular weight plant DNA. Nucleic Acids Res. 8:4321-4326.

Nandi, M., Macdonald, J., Liu, P., Weselowski, B., and Yuan, Z. 2018. Clavibacter michiganensis ssp. michiganensis: Bacterial canker of tomato, molecular interactions and disease management. Mol. Plant Pathol. 19:2036-2050.

Peritore-Galve, F. C., Tancos, M. A., and Smart, C. D. 2021. Bacterial canker of tomato: Revisiting a global and economically damaging seedborne pathogen. Plant Dis. Published online.

Ponciano, G., Ishihara, H., Tsuyumu, S., and Leach, J. E. 2003. Bacterial effectors in plant disease and defense: Keys to durable resistance? Plant Dis. 87:1272-1282.

R Core Team. 2019. R: A Language and Environment for Statistical Computing. R Foundation for Statistical Computing, Vienna, Austria.

Richardson, E. J., and Watson, M. 2013. The automatic annotation of bacterial genomes. Brief. Bioinform. 14:1-12.

Savory, E. A., Weisberg, A. J., Stevens, D. M., Creason, A. L., Fuller, S. L., Pearce, E. M., and Chang, J. H. 2020. Phytopathogenic Rhodococcus have diverse plasmids with few conserved virulence functions. Front. Microbiol. 11:1022.

Stork, I., Gartemann, K., Burger, A., and Eichenlaub, R. 2008. A family of serine proteases of Clavibacter michiganensis subsp. michiganensis chpC plays a role in colonization of the host plant tomato. Mol. Plant Pathol. 9:599-608.

Syverson, R. L. 2011. Multiple approaches towards understanding virulence in Clavibacter michiganensis subsp. sepedonicus, causal agent of bacterial ring rot of potato. Ph.D. dissertation. University of Minnesota, St. Paul, MN, U.S.A.

Tambong, J. T. 2017. Comparative genomics of Clavibacter michiganensis subspecies, pathogens of important agricultural crops. PLoS One 12: 0172295.

Tancos, M. A., Chalupowicz, L., Barash, I., Manulis-Sasson, S., and Smart, C. D. 2013. Tomato fruit and seed colonization by Clavibacter michiganensis subsp. michiganensis through external and internal routes. Appl. Environ. Microbiol. 79:6948-6957.

Thapa, S. P., Davis, E. W., II, Lyu, Q., Weisberg, A. J., Stevens, D. M. Clarke, C. R., Coaker, G., and Chang, J. H. 2019. The evolution, ecology, and mechanisms of infection by gram-positive, plant-associated bacteria. Annu. Rev. Phytopathol. 57:341-365.

Thapa, S. P., Pattathil, S., Hahn, M., Jacques, M.-A., Gilbertson, B., and Coaker, G. 2017. Genomic analysis of Clavibacter michiganensis reveals insight into virulence strategies and genetic diversity of a gram-positive bacterial pathogen. Mol. Plant-Microbe Interact. 30:786802.

van der Geize, R., de Jong, W., Hessels, G. I., Grommen, A. W. F., Jacobs, A. A. C., and Dijkhuizen, L. 2008. A novel method to generate unmarked gene deletions in the intracellular pathogen Rhodococcus equi using 5-fluorocytosine conditional lethality. Nucleic Acids Res. 36:e151.

Xie, Z., and Tang, H. 2017. ISEScan: Automated identification of insertion sequence elements in prokaryotic genomes. Bioinformatics 33: 3340-3347. 